\title{
Extension of point-finite partitions of unity
}

\author{
by \\ Haruto Ohta (Shizuoka) and Kaori Yamazaki (Ibaraki)
}

\begin{abstract}
A subspace $A$ of a topological space $X$ is said to be $P^{\gamma}$-embedded ( $P^{\gamma}$ (pointfinite)-embedded) in $X$ if every (point-finite) partition of unity $\alpha$ on $A$ with $|\alpha| \leq \gamma$ extends to a (point-finite) partition of unity on $X$. The main results are: (Theorem A) A subspace $A$ of $X$ is $P^{\gamma}$ (point-finite)-embedded in $X$ iff it is $P^{\gamma}$-embedded and every countable intersection $B$ of cozero-sets in $X$ with $B \cap A=\emptyset$ can be separated from $A$ by a cozero-set in $X$. (Theorem B) The product $A \times[0,1]$ is $P^{\gamma}$ (point-finite)-embedded in $X \times[0,1]$ iff $A \times Y$ is $P^{\gamma}$ (point-finite)-embedded in $X \times Y$ for every compact Hausdorff space $Y$ with $w(Y) \leq \gamma$ iff $A$ is $P^{\gamma}$-embedded in $X$ and every subset $B$ of $X$ obtained from zero-sets by means of the Suslin operation, with $B \cap A=\emptyset$, can be separated from $A$ by a cozero-set in $X$. These characterizations are used to answer certain questions of Dydak. In particular, it is shown that, assuming $\mathrm{CH}$, the property of $A \times[0,1]$ to be $P^{\gamma}$ (point-finite)-embedded in $X \times[0,1]$ is stronger than that of $A$ being $P^{\gamma}$ (point-finite)-embedded in $X$.
\end{abstract}

1. Introduction. By a space we mean a topological space. A partition of unity $\alpha$ on a space $X$ is called point-finite (resp. locally finite) if the family $\{\operatorname{coz}(f): f \in \alpha\}$ is point-finite (resp. locally finite) in $X$, where $\operatorname{coz}(f)=\{x \in X: f(x) \neq 0\}$. Let $A$ be a subspace of a space $X$ and $\gamma$ an infinite cardinal. When $\alpha=\left\{f_{\lambda}\right\}$ and $\beta=\left\{g_{\lambda}\right\}$ are partitions of unity on $A$ and $X$, respectively, we say that $\beta$ is an extension of $\alpha$ if $f_{\lambda}=\left.g_{\lambda}\right|_{A}$ for each $\lambda$. Dydak [3] defined $A$ to be $P^{\gamma}$ (point-finite)-embedded (resp. $P^{\gamma}$ (locally finite)embedded) in $X$ if every point-finite (resp. locally finite) partition of unity $\alpha$ on $A$, with $|\alpha| \leq \gamma$, extends to a point-finite (resp. locally finite) partition of unity on $X$. Extensive studies of $P^{\gamma}$ (locally finite)-embedding have been made by Dydak [3], [4] and the second author [21] and [23].

2000 Mathematics Subject Classification: Primary 54C20; Secondary 54C55, 54H05.

Key words and phrases: partition of unity, extension, product, analytic set, $P$ embedding, $P$ (point-finite)-embedding, $M$-embedding, $\sigma$-complete, AR.

Research of the second author is partially supported by Grand-in-Aid for the Encouragement of Young Scientists (B) (No. 16740028) of Ministry of Education, Science, Sports and Culture. 
In this paper, we consider $P^{\gamma}$ (point-finite)-embeddings. In particular, we prove Theorems A and B stated in the abstract and apply them to answer Dydak's questions concerning $P^{\gamma}$ (point-finite)-embeddings stated below.

Recall from [3] that $A$ is $P^{\gamma}$-embedded in $X$ if every partition of unity $\alpha$ on $A$ with $|\alpha| \leq \gamma$ extends to a partition of unity on $X$ (see [2], [19] for the original definition of $P^{\gamma}$-embedding). Recall from [18] that $A$ is $M^{\gamma}$ embedded in $X$ if for every AR $Y$ with $w(Y) \leq \gamma$, every continuous map from $A$ to $Y$ extends continuously over $X$. It is known (see [3]) that these extension properties are related as follows, where $A \rightarrow B$ means that every $A$-embedded subspace is $B$-embedded:

$$
M^{\gamma} \rightarrow P^{\gamma} \text { (point-finite) } \rightarrow P^{\gamma} \stackrel{(*)}{\longleftarrow} P^{\gamma} \text { (locally finite) } .
$$

Przymusiński-Wage [15] showed that the arrow $(*)$ cannot be reversed by giving an example of a collectionwise normal space $Z$ having a closed subspace which is not $P^{\omega}$ (locally finite)-embedded, and Dydak [3] showed that the implication " $P^{\gamma}$ (locally finite) $\rightarrow P^{\gamma}$ (point-finite)" is not true in general (see also [24]). In Section 3, we give an example of a subspace which is $P^{\gamma}$ (point-finite)-embedded for every $\gamma$ but not $M^{\omega}$-embedded (Example 3.4 ), and prove that every closed subspace of the space $Z$ of PrzymusińskiWage mentioned above is $M^{\gamma}$-embedded for every $\gamma$ (Example 3.8). These results answer Dydak's questions [3, Problems 12.10 and 12.11] negatively. Moreover, Dydak [3, Problem 13.6] asked: If $A$ is $P^{\gamma}$ (point-finite)-embedded in $X$, is then $A \times[0,1] P^{\gamma}$ (point-finite)-embedded in $X \times[0,1]$ ? It is known that the answers to the similar questions for $P^{\gamma}{ }_{-}, M^{\gamma}$ - and $P^{\gamma}$ (locally finite)embeddings are all positive (see Alò-Sennott [1], Sennott [18] and Yamazaki [21], respectively). As an application of Theorem B, we show that the answer is negative for $P^{\gamma}$ (point-finite)-embeddings under the assumption of the continuum hypothesis (Examples 3.5 and 3.7).

For a set $A,|A|$ denotes the cardinality of $A$. As usual, a cardinal is an initial ordinal and an ordinal is identified with the set of smaller ordinals. Let $\omega$ denote the first infinite cardinal and $\omega_{1}$ the first uncountable cardinal. Our terminology and notation follow [5] and [13].

2. $P^{\gamma}$ (point-finite)-embeddings and products. A zero-set in a space $X$ is a set of the form $f^{-1}(0)$ for some real-valued continuous function $f$ on $X$ and a cozero-set is the complement of a zero-set. For a space $X$, let $\mathcal{Z}(X)$ (resp. $\mathcal{C} o z(X))$ denote the family of all zero-sets (resp. cozero-sets) in $X$. A set $A \subseteq X$ is called a Suslin- $\mathcal{Z}$-set in $X$ if there exists a family $\left\{Z_{\sigma}: \sigma \in{ }^{<\omega} \omega\right\} \subseteq \mathcal{Z}(X)$ such that $A=\bigcup_{t \in \omega_{\omega}} \bigcap_{n<\omega} Z_{t \mid n}$, where ${ }^{\alpha} \omega$ denotes the set of all maps from $\alpha$ to $\omega$ and ${ }^{<\omega} \omega=\bigcup_{n<\omega}{ }^{n} \omega$ (see [16]). All Baire sets, i.e., members of the smallest $\sigma$-algebra including $\mathcal{Z}(X)$, are Suslin- $\mathcal{Z}$-sets. As usual, we call a Suslin- $\mathcal{Z}$-set and a Baire set in a metric space an analytic 
set and a Borel set, respectively. Now, we consider the following conditions on a subspace $A$ of a space $X$ :

$\left(b_{1}\right)$ For every Suslin- $\mathcal{Z}$-set $B$ in $X$ with $B \cap A=\emptyset$, there exists $U \in$ $\mathcal{C}$ oz $(X)$ such that $B \subseteq U$ and $U \cap A=\emptyset$.

$\left(b_{2}\right)$ For every Baire set $B$ in $X$ with $B \cap A=\emptyset$, there exists $U \in \mathcal{C}$ oz $(X)$ such that $B \subseteq U$ and $U \cap A=\emptyset$.

$\left(b_{3}\right)$ For every countable family $\left\{G_{n}: n<\omega\right\} \subseteq \mathcal{C}$ oz $(X)$ with $\bigcap_{n<\omega} G_{n}$ $\cap A=\emptyset$, there exists $U \in \mathcal{C}$ oz $(X)$ such that $\bigcap_{n<\omega} G_{n} \subseteq U$ and $U \cap A=\emptyset$.

Evidently, $\left(b_{1}\right)$ implies $\left(b_{2}\right)$ and $\left(b_{2}\right)$ implies $\left(b_{3}\right)$ (see Remark 2.8 below). Now, we prove the theorems announced in the abstract.

THEOREM 2.1. Let $A$ be a subspace of a space $X$ and $\gamma$ an infinite cardinal. Then the following are equivalent:

(1) $A$ is $P^{\gamma}$ (point-finite)-embedded in $X$,

(2) $A$ is $P^{\gamma}$-embedded in $X$ and $P^{\omega}$ (point-finite)-embedded in $X$,

(3) $A$ is $P^{\gamma}$-embedded in $X$ and satisfies $\left(b_{3}\right)$ in $X$.

Proof. $(1) \Rightarrow(2)$ : Obvious. $(2) \Rightarrow(3)$ : To prove that $A$ satisfies $\left(b_{3}\right)$ in $X$, take a countable family $\left\{G_{n}: n<\omega\right\} \subseteq \mathcal{C}$ oz $(X)$ with $\bigcap_{n<\omega} G_{n} \cap A=\emptyset$. We may assume that $G_{n+1} \subseteq G_{n}$ for each $n<\omega$ and $G_{0}=X$. Take continuous functions $f_{n}: X \rightarrow\left[0,1 / 2^{n}\right], n<\omega$, with $G_{n}=\operatorname{coz}\left(f_{n}\right)$, and define $f=$ $\sum_{n<\omega} f_{n}$. Note that $f(x)>0$ for all $x \in X$. For each $n<\omega$, define a function $f_{n}^{*}: A \rightarrow[0,1]$ by $f_{n}^{*}(x)=f_{n}(x) / f(x)$ for $x \in A$. Then $\left\{f_{n}^{*}: n<\omega\right\}$ is a point-finite partition of unity on $A$. Since $A$ is $P^{\omega}$ (point-finite)-embedded in $X$, there exists a point-finite partition of unity $\left\{g_{n}: n<\omega\right\}$ on $X$ such that $\left.g_{n}\right|_{A}=f_{n}^{*}$ for each $n<\omega$. Let

$$
U=\bigcup_{n<\omega}\left\{x \in X: g_{n}(x) \cdot f(x) \neq f_{n}(x)\right\} .
$$

Then $U \in \operatorname{Coz}(X), \bigcap_{n<\omega} G_{n} \subseteq U$ since $\left\{g_{n}: n<\omega\right\}$ is point-finite, and $U \cap A=\emptyset$. Hence, $A$ satisfies $\left(b_{3}\right)$ in $X$.

$(3) \Rightarrow(1)$ : Let $\alpha=\left\{f_{\lambda}: \lambda \in \Lambda\right\}$ be a point-finite partition of unity on $A$ with $|\Lambda| \leq \gamma$. Since $A$ is $P^{\gamma}$-embedded in $X, \alpha$ extends to a partition of unity $\beta=\left\{g_{\lambda}: \lambda \in \Lambda\right\}$ on $X$. Let $B=\{x \in X: \beta$ is not point-finite at $x\}$. We show that $B$ is the countable intersection of cozero-sets in $X$. For each $n<\omega$ and each $x \in X$, define

$$
k_{n}(x)=\max \left\{\sum_{\lambda \in \delta} g_{\lambda}(x): \delta \subseteq \Lambda,|\delta| \leq n\right\} .
$$

Since $\beta$ is a partition of unity, the functions $k_{n}: X \rightarrow[0,1], n<\omega$, are continuous, and for each $x \in X,\left|\left\{\lambda \in \Lambda: g_{\lambda}(x)>0\right\}\right| \leq n$ if and only if $k_{n}(x)=1$. This implies that $X \backslash B=\bigcup_{n<\omega} k_{n}^{-1}(1)$, and hence, $B$ is the 
intersection of countably many cozero-sets in $X$. Since $B \cap A=\emptyset$ and $A$ satisfies $\left(b_{3}\right)$ in $X$, we can find a continuous function $h: X \rightarrow[0,1]$ such that $B \subseteq \operatorname{coz}(h)$ and $\operatorname{coz}(h) \cap A=\emptyset$. For each $\lambda \in \Lambda$, define a function $g_{\lambda}^{*}$ on $X$ by $g_{\lambda}^{*}(x)=\max \left\{g_{\lambda}(x)-h(x), 0\right\}$ for $x \in X$. Then $\left\{\operatorname{coz}\left(g_{\lambda}^{*}\right): \lambda \in \Lambda\right\}$ is point-finite in $X$, because if $h(x)=0$, then $g_{\lambda}^{*}(x)=g_{\lambda}(x)$ and $x \notin B$; and if $h(x)>0$, then only finitely many $g_{\lambda}$ 's exceed $h$ at $x$ since $\sum_{\lambda \in \Lambda} g_{\lambda}(x)=1$. Since $g_{\lambda}^{*}(x) \leq g_{\lambda}(x)$ for each $\lambda \in \Lambda$ and each $x \in X$, it follows from [4, Corollary 2.6] that the function $\sum_{\lambda \in \Lambda} g_{\lambda}^{*}$ is continuous. Fix an arbitrary $\mu \in \Lambda$ and define

$$
g_{\mu}^{* *}(x)=g_{\mu}^{*}(x)+1-\sum_{\lambda \in \Lambda} g_{\lambda}^{*}(x) \quad \text { for } x \in X .
$$

Finally, putting $g_{\lambda}^{* *}=g_{\lambda}^{*}$ for each $\lambda \in \Lambda \backslash\{\mu\}$, we obtain a point-finite partition of unity $\left\{g_{\lambda}^{* *}: \lambda \in \Lambda\right\}$ on $X$ extending $\alpha$. Hence, $A$ is $P^{\gamma}$ (point-finite)embedded in $X$.

We turn to considering the problem when $A \times Y$ is $P^{\gamma}$ (point-finite)embedded in $X \times Y$ for all (or certain) compact Hausdorff spaces $Y$. We need the following result due to Alò and Sennott [1] as a lemma.

Lemma 2.2 (Alò-Sennott). Let $A$ be a $P^{\gamma}$-embedded subspace of a space $X$, where $\gamma$ is an infinite cardinal. Then $A \times Y$ is $P^{\gamma}$-embedded in $X \times Y$ for every compact Hausdorff space $Y$ with $w(Y) \leq \gamma$.

Since the countable union of cozero-sets is a cozero-set, we have the following corollary from Theorem 2.1 and Lemma 2.2.

Corollary 2.3. Let $A$ be a $P^{\gamma}$ (point-finite)-embedded subspace of a space $X$, where $\gamma$ is an infinite cardinal. Then $A \times Y$ is $P^{\gamma}$ (point-finite)embedded in $X \times Y$ for every countable, compact metric space $Y$.

The next lemma is well known, but we can find no good reference.

Lemma 2.4 (folklore). Let $X$ and $Y$ be spaces and $\operatorname{pr}_{X}: X \times Y \rightarrow X$ the projection.

(1) If $Y$ is separable, then $\operatorname{pr}_{X}$ carries cozero-sets to cozero-sets.

(2) If $Y$ is compact, then $\operatorname{pr}_{X}$ carries cozero-sets to cozero-sets and carries zero-sets to zero-sets.

(3) If $Y$ is compact, then $\operatorname{pr}_{X}$ carries Suslin-Z-zets to Suslin $\mathcal{Z}$-sets.

Proof. (1) Let $D$ be a countable dense set in $Y$. Then, for every cozero-set $G$ in $X \times Y, \operatorname{pr}_{X}[G]=\bigcup_{y \in D}\{x \in X:\langle x, y\rangle \in G\} \in \operatorname{Coz}(X)$.

(2) This follows from the fact that if $Y$ is compact, then for every realvalued continuous function $h$ on $X \times Y$, the functions $f$ and $g$ on $X$ defined by $f(x)=\sup \{h(x, y): y \in Y\}$ and $g(x)=\inf \{h(x, y): y \in Y\}$ for $x \in X$ are continuous (see [6, Lemma 1.1]). 
(3) This is a consequence of (2) since we can assume that $Z_{t \mid n} \subseteq Z_{t \mid m}$ whenever $m<n$ in the definition of a Suslin- $\mathcal{Z}$-set.

The next lemma is due to the referee's suggestion.

Lemma 2.5. For every Suslin-Z-Z -set $B$ in a space $X$, there exists a continuous map $f: X \rightarrow Q$, where $Q$ is the Hilbert cube, such that $B=f^{-1}[S]$ for some analytic set $S$ in $Q$.

Proof. This is a consequence of the following observation: For any collection of countably many zero-sets $Z_{n}=f_{n}^{-1}(0), n<\omega$, in $X$, consider the diagonal map $f=\triangle_{n<\omega} f_{n}: X \rightarrow Q$. Then each $Z_{n}$ is the inverse image of a closed set in $Q$.

Now, combining Lemma 2.5 with Theorem 2.1, Lemmas 2.2 and 2.4, we have the following theorem.

Theorem 2.6. Let $A$ be a subspace of a space $X$ and $\gamma$ an infinite cardinal. Then the following are equivalent:

(1) $A \times Y$ is $P^{\gamma}$ (point-finite)-embedded in $X \times Y$ for every compact Hausdorff space $Y$ with $w(Y) \leq \gamma$,

(2) $A \times[0,1]$ is $P^{\gamma}($ point-finite $)$-embedded in $X \times[0,1]$,

(3) $A \times Y$ is $P^{\gamma}$ (point-finite)-embedded in $X \times Y$ for some uncountable, compact metric space $Y$,

(4) $A$ is $P^{\gamma}$-embedded in $X$ and satisfies $\left(b_{1}\right)$ in $X$.

Proof. (1) $\Rightarrow(2) \Rightarrow(3)$ : Obvious. (3) $\Rightarrow(4)$ : Assume that $A \times Y$ is $P^{\gamma}$ (pointfinite)-embedded in $X \times Y$ for some uncountable, compact metric space $Y$. As every $P^{\gamma}$ (point-finite)-embedded subspace is $P^{\gamma}$-embedded, it suffices to show that $A$ satisfies $\left(b_{1}\right)$ in $X$. Let $B$ be a Suslin- $\mathcal{Z}$-set in $X$ with $B \cap A=\emptyset$. Then, by Lemma 2.5 , there exists a continuous map $f: X \rightarrow Q$ such that $B=f^{-1}[S]$ for some analytic set $S$ in $Q$. It is known that $S$ is the projection of a $G_{\delta^{-} \text {set }} G$ in $Q \times \mathbb{K}$, where $\mathbb{K}$ is the Cantor set (see [11]). Since $\mathbb{K}$ can be embedded in $Y$, we regard $G$ as a $G_{\delta^{-}}$set in $Q \times Y$. Put $H=\left(f \times \operatorname{id}_{Y}\right)^{-1}[G]$, where $\operatorname{id}_{Y}$ is the identity of $Y$. Then $H$ is the intersection of countably many cozero-sets in $X \times Y$ and $B=\operatorname{pr}_{X}[H]$. Since $A \times Y$ satisfies $\left(b_{3}\right)$ in $X \times Y$ by Theorem 2.1, there exists $U \in \mathcal{C} O z(X \times Y)$ such that $H \subseteq U$ and $U \cap(A \times Y)=\emptyset$. Finally, put $V=\operatorname{pr}_{X}[U]$. Then $V \in \mathcal{C} o z(X)$ by Lemma 2.4(2), $B \subseteq V$ and $V \cap A=\emptyset$. Hence, $A$ satisfies $\left(b_{1}\right)$ in $X$.

$(4) \Rightarrow(1)$ : Let $Y$ be a compact Hausdorff space with $w(Y) \leq \gamma$. By Theorem 2.1 and Lemma 2.2, it suffices to show that $A \times Y$ satisfies $\left(b_{3}\right)$ in $X \times Y$. Let $B$ be the intersection of countably many cozero-sets in $X \times Y$ with $B \cap(A \times Y)=\emptyset$. Then it follows from Lemma 2.4(3) that $\operatorname{pr}_{X}[B]$ is a Suslin- $\mathcal{Z}$-set in $X$ with $B \cap A=\emptyset$. Since $A$ satisfies $\left(b_{1}\right)$ in $X$, there exists $U \in \operatorname{Coz}(X)$ such that $\operatorname{pr}_{X}[B] \subseteq U$ and $U \cap A=\emptyset$. Putting $V=U \times Y$, 
we obtain $V \in \mathcal{C} O z(X \times Y)$ such that $B \subseteq V$ and $V \cap(A \times Y)=\emptyset$. Hence, $A \times Y$ satisfies $\left(b_{3}\right)$ in $X \times Y$.

The reader might ask if "compact metric" can be replaced by "compact Hausdorff" in condition (3) of Theorem 2.6. In Remark 3.6 below, we show that metrizability of $Y$ is essential in this condition.

The following corollary can be proved similarly to $(3) \Rightarrow(4)$ in Theorem 2.6 if we use Lemma 2.4(1) and the fact that every analytic set in $Q$ is the projection of a zero-set in $Q \times \mathbb{P}$, where $\mathbb{P}$ is the space of irrational numbers (see [11]).

Corollary 2.7. Let $A$ be a $P^{\gamma}$-embedded closed subspace of a space $X$, where $\gamma$ is an infinite cardinal, and assume that either $X \times \mathbb{P}$ is normal or $(X \backslash A) \times \mathbb{P}$ is Lindelöf. Then $A \times Y$ is $P^{\gamma}($ point-finite $)$-embedded in $X \times Y$ for every compact Hausdorff space $Y$ with $w(Y) \leq \gamma$.

In the remaining part of this section, we consider the relationship between conditions $\left(b_{i}\right), i=1,2,3$, and the following conditions, from the literature, on a subspace $A$ of a space $X$.

$\left(a_{\gamma}\right)$ For every $\gamma$-separable continuous pseudometric $\varrho$ on $X$, there exists $F \in \mathcal{Z}(X)$ such that $A \subseteq F \subseteq\{x \in X:(\exists y \in A)(\varrho(x, y)=0)\}$.

(c) For every $B \in \mathcal{Z}(X)$ with $B \cap A=\emptyset$, there exists $U \in \mathcal{C} o z(X)$ such that $B \subseteq U$ and $U \cap A=\emptyset$.

Here, a pseudometric $d$ on a space $X$ is called $\gamma$-separable if the weight of the pseudometric space $(X, d)$ is not greater than $\gamma$. Sennott [18] proved that $A$ is $M^{\gamma}$-embedded in $X$ if and only if $A$ is $P^{\gamma}$-embedded in $X$ and satisfies $\left(a_{\gamma}\right)$ in $X$. On the other hand, it is known (see [8, Theorem 1.18]) that $A$ satisfies $(c)$ in $X$ if every real-valued continuous function on $A$ extends continuously over $X$, or equivalently, $A$ is $P^{\omega}$-embedded in $X$ (see [7] and [9]). Obviously, $\left(b_{3}\right)$ implies $(c)$.

Proposition 2.8. $\left(a_{\omega}\right)$ implies $\left(b_{1}\right)$.

Proof. Assume that a subspace $A$ of a space $X$ satisfies $\left(a_{\omega}\right)$ in $X$. Let $B$ be a Suslin- $\mathcal{Z}$-set in $X$ with $B \cap A=\emptyset$. Then, by Lemma 2.5 , there exists a continuous map $f$ from $X$ to the Hilbert cube $Q$ such that $B=f^{-1}[f[B]]$. Let $d$ be the metric on $Q$, and define $\varrho(x, y)=d(f(x), f(y))$ for $x, y \in X$. Then $\varrho$ is an $\omega$-separable continuous pseudometric on $X$ such that $\{x \in X$ : $(\exists y \in A)(\varrho(x, y)=0)\} \cap B=\emptyset$. Hence, by $\left(a_{\omega}\right)$, we can find $U \in \operatorname{Coz}(X)$ such that $B \subseteq U$ and $U \cap A=\emptyset$.

REMARK 2.9. Summing up the above observations, we have the implications

$$
\left(a_{\omega}\right) \Rightarrow\left(b_{1}\right) \Rightarrow\left(b_{2}\right) \Rightarrow\left(b_{3}\right) \Rightarrow(c)
$$


In the next section, we give examples showing that $\left(b_{1}\right) \nRightarrow\left(a_{\omega}\right)$ and $(c) \nRightarrow$ $\left(b_{3}\right)$, and show that $\left(b_{3}\right) \nRightarrow\left(b_{2}\right)$ and $\left(b_{2}\right) \nRightarrow\left(b_{1}\right)$ assuming the continuum hypothesis.

3. Examples. As stated in the introduction, we now apply Theorems 2.1 and 2.6 to answer Dydak's questions [3, Problems 12.10, 12.11 and 13.6]. Throughout this section, $\mathbb{R}$ denotes the set of real numbers endowed with the Euclidean topology $\tau$. When $A \subseteq X \subseteq \mathbb{R}, X_{A}$ denotes the space with the underlying set $X$ and with the topology $\left\{U \cup K: U \in \tau_{X}, K \subseteq X \backslash A\right\}$, where $\tau_{X}$ is the subspace topology on $X$ induced from $\tau$. It is known that $X_{A}$ is a paracompact Hausdorff space (see [5, Example 5.1.22]). We begin by determining when the subspace $A$ satisfies $\left(b_{i}\right), i=1,2,3$, in $X_{A}$ in terms of subsets of $\mathbb{R}$.

Lemma 3.1. Let $A \subseteq X \subseteq \mathbb{R}$ and $S \subseteq X$. Then:

(1) $S$ is a cozero-set in $X_{A}$ if and only if there exist an open set $U$ in $\mathbb{R}$ and an $F_{\sigma}$-set $F$ in $\mathbb{R}$ such that $S \cap A \subseteq U \cap X \subseteq S, S \subseteq F$ and $F \cap A=S \cap A$.

(2) If $S$ is a Baire set in $X_{A}$, then there exists a Borel set $B$ in $\mathbb{R}$ such that $S \subseteq B$ and $B \cap A=S \cap A$.

(3) If $S$ is a Suslin-Z-set in $X_{A}$, then there exists an analytic set $B$ in $\mathbb{R}$ such that $S \subseteq B$ and $B \cap A=S \cap A$.

Proof. (1) First, observe that (i) a set $S \subseteq X$ is open in $X_{A}$ if and only if there exists an open set $U$ in $\mathbb{R}$ with $S \cap A \subseteq U \cap X \subseteq S$, and (ii) a set $S \subseteq X$ is closed in $X_{A}$ if and only if there exists a closed set $F$ in $\mathbb{R}$ such that $S \subseteq F$ and $S \cap A=F \cap A$. Now, (1) follows from (i) and (ii) since, by the normality of $X_{A}, S \in \mathcal{C} O z\left(X_{A}\right)$ if and only if $S$ is an open $F_{\sigma}$-set in $X_{A}$.

(2) Let $\mathcal{S}$ be the family of all sets $S \subseteq X$ such that there exist Borel sets $B$ and $B^{\prime}$ in $\mathbb{R}$ such that $S \cap A \subseteq B \cap X \subseteq S, S \subseteq B^{\prime}$ and $B^{\prime} \cap A=S \cap A$. Then $\mathcal{S}$ is a $\sigma$-algebra of subsets of $X$, and $\mathcal{C} o z\left(X_{A}\right) \subseteq \mathcal{S}$ by (1). Hence, all Baire sets in $X_{A}$ belong to $\mathcal{S}$, from which (2) can be deduced.

(3) If $S$ is a Suslin- $\mathcal{Z}$-set in $X_{A}$, then there exists $\left\{Z_{\sigma}: \sigma \in<\omega \omega\right\} \subseteq$ $\mathcal{Z}\left(X_{A}\right)$ such that $S=\bigcup_{t \in \omega_{\omega}} \bigcap_{n<\omega} Z_{t \mid n}$. Define $B=\bigcup_{t \in \omega_{\omega}} \bigcap_{n<\omega} \operatorname{cl}_{\mathbb{R}} Z_{t \mid n}$. Then $B$ is an analytic set in $\mathbb{R}$ with $S \subseteq B$. Since $\operatorname{cl}_{\mathbb{R}} Z_{\sigma} \cap A=Z_{\sigma} \cap A$ for each $\sigma \in{ }^{<\omega} \omega, B \cap A=S \cap A$.

Proposition 3.2. Let $A \subseteq X \subseteq \mathbb{R}$. Then:

(1) A satisfies $\left(b_{1}\right)$ in $X_{A}$ if and only if for every analytic set $B$ in $\mathbb{R}$ with $B \cap A=\emptyset$, there exists an $F_{\sigma}$-set $F$ in $\mathbb{R}$ such that $B \cap X \subseteq F$ and $F \cap A=\emptyset$.

(2) A satisfies $\left(b_{2}\right)$ in $X_{A}$ if and only if for every Borel set $B$ in $\mathbb{R}$ with $B \cap A=\emptyset$, there exists an $F_{\sigma}$-set $F$ in $\mathbb{R}$ such that $B \cap X \subseteq F$ and $F \cap A=\emptyset$. 
(3) A satisfies $\left(b_{3}\right)$ in $X_{A}$ if and only if for every countable family $\left\{F_{n}\right.$ : $n<\omega\}$ of $F_{\sigma}$-sets in $\mathbb{R}$ such that $\bigcap_{n<\omega} F_{n} \cap A=\emptyset$ and $F_{n} \cap A$ is open in $A$ for each $n<\omega$, there exists an $F_{\sigma}$-set $F$ in $\mathbb{R}$ such that $\bigcap_{n<\omega} F_{n} \cap X \subseteq F$ and $F \cap A=\emptyset$.

Proof. (1) Assume that $A$ satisfies $\left(b_{1}\right)$ in $X_{A}$ and let $B$ be an analytic set in $\mathbb{R}$ with $B \cap A=\emptyset$. Since the topology of $\mathbb{R}_{A}$ is finer than that of $\mathbb{R}, B$ is a Suslin- $\mathcal{Z}$-set in $\mathbb{R}_{A}$, and hence, $B \cap X$ is also a Suslin- $\mathcal{Z}$-set in $X_{A}$. Thus, it follows from $\left(b_{1}\right)$ that there exists $U \in \mathcal{C} O z\left(X_{A}\right)$ such that $B \cap X \subseteq U$ and $U \cap A=\emptyset$. By Lemma 3.1(1), there exists an $F_{\sigma}$-set $F$ in $\mathbb{R}$ such that $U \subseteq F$ and $F \cap A=U \cap A$. Then $B \cap X \subseteq F$ and $F \cap A=\emptyset$. Conversely, assume that $A$ satisfies the latter condition in (1) and let $C$ be a Suslin- $\mathcal{Z}$-set in $X_{A}$ with $C \cap A=\emptyset$. Then, by Lemma 3.1(3), there exists an analytic set $H$ in $\mathbb{R}$ such that $C \subseteq H$ and $H \cap A=\emptyset$. By the assumption, we can find an $F_{\sigma}$-set $F$ in $\mathbb{R}$ such that $H \cap X \subseteq F$ and $F \cap A=\emptyset$. Since $F \cap X \in \operatorname{Coz}\left(X_{A}\right)$ by Lemma $3.1(1), A$ satisfies $\left(b_{1}\right)$ in $X_{A}$. (2) can be proved similarly to (1) using Lemma 3.1(2) instead of Lemma 3.1(3).

(3) Assume that $A$ satisfies $\left(b_{3}\right)$ in $X_{A}$ and let $\left\{F_{n}: n<\omega\right\}$ be a countable family of $F_{\sigma}$-sets in $\mathbb{R}$ such that $\bigcap_{n<\omega} F_{n} \cap A=\emptyset$ and $F_{n} \cap A$ is open in $A$ for each $n<\omega$. For each $n<\omega$, since $X_{A}$ is normal, we can find $E_{n} \in \operatorname{Coz}\left(X_{A}\right)$ such that $F_{n} \cap X \subseteq E_{n}$ and $E_{n} \cap A=F_{n} \cap A$. Since $\bigcap_{n<\omega} E_{n} \cap A=\emptyset$, it follows from $\left(b_{3}\right)$ that there exists $U \in \operatorname{Coz}\left(X_{A}\right)$ such that $\bigcap_{n<\omega} E_{n} \subseteq U$ and $U \cap A=\emptyset$. By Lemma 3.1(1), there exists an $F_{\sigma}$-set $F$ in $\mathbb{R}$ such that $U \subseteq F$ and $F \cap A=U \cap A$. Then

$$
\bigcap_{n<\omega} F_{n} \cap X \subseteq \bigcap_{n<\omega} E_{n} \subseteq U \subseteq F
$$

and $F \cap A=\emptyset$. Conversely, assume that $A$ satisfies the latter condition in (3), and take $\left\{G_{n}: n<\omega\right\} \subseteq \mathcal{C}$ oz $\left(X_{A}\right)$ such that $\bigcap_{n<\omega} G_{n} \cap A=\emptyset$. For each $n<\omega$, by Lemma 3.1(1), there exists an $F_{\sigma}$-set $H_{n}$ in $\mathbb{R}$ such that $G_{n} \subseteq H_{n}$ and $H_{n} \cap A=G_{n} \cap A$. Since $\bigcap_{n<\omega} H_{n} \cap A=\emptyset$ and $H_{n} \cap A$ is open in $A$ for each $n<\omega$, it follows from our assumption that there exists an $F_{\sigma}$-set $H$ in $\mathbb{R}$ such that $\bigcap_{n<\omega} H_{n} \cap X \subseteq H$ and $H \cap A=\emptyset$. Then $H \cap X \in \operatorname{Coz}\left(X_{A}\right)$ by Lemma 3.1(1), $\bigcap_{n<\omega} G_{n} \subseteq H \cap X$ and $(H \cap X) \cap A=\emptyset$. Hence, $A$ satisfies $\left(b_{3}\right)$ in $X_{A}$.

Now, we are in a position to construct examples. A subspace $A$ of a space $X$ is said to be $P$-embedded in $X$ if it is $P^{\gamma}$-embedded in $X$ for every $\gamma . M$ and $P$ (point-finite)-embeddings are defined similarly. If $A \subseteq X \subseteq \mathbb{R}$, then the closed subspace $A$ of $X_{A}$ is always $P$-embedded in $X_{A}$, since $X_{A}$ is paracompact. The last statement of the following example was proved by the second author in [24]; however, now it is an immediate consequence of Proposition 3.2(3) and Theorem 2.1. 
EXAmple 3.3. Let $\mathbb{Q}$ be the set of rational numbers. Then $\mathbb{Q}$ fails to satisfy $\left(b_{3}\right)$ in $\mathbb{R}_{\mathbb{Q}}$. Hence, $\mathbb{Q}$ is not $P^{\omega}$ (point-finite)-embedded in $\mathbb{R}_{\mathbb{Q}}$.

Example 3.3 shows that $(c) \nRightarrow\left(b_{3}\right)$ in general. Recall from $[5,5.5 .4]$ that there exists a set $A \subseteq \mathbb{R}$, called a Bernstein set, such that every compact set in $\mathbb{R}$ contained in either $A$ or $\mathbb{R} \backslash A$ is countable.

Example 3.4. Let $A$ be a Bernstein set in $\mathbb{R}$. Then $A$ satisfies $\left(b_{1}\right)$ in $\mathbb{R}_{A}$ but fails to satisfy $(a)_{\omega}$ in $\mathbb{R}_{A}$. Hence, $A \times Y$ is $P$ (point-finite)-embedded in $\mathbb{R}_{A} \times Y$ for every compact Hausdorff space $Y$, but $A$ is not $M^{\omega}$-embedded in $\mathbb{R}_{A}$.

Proof. Let $B$ be an analytic set in $\mathbb{R}$ with $B \cap A=\emptyset$. Then $B$ must be countable, since every uncountable analytic set in $\mathbb{R}$ contains a Cantor set (see [10, Theorem 94]). By Proposition 3.2(1), this implies that $A$ satisfies $\left(b_{1}\right)$ in $\mathbb{R}_{A}$. On the other hand, the Euclidean metric $d$ on $\mathbb{R}$ is an $\omega$-separable continuous pseudometric on $\mathbb{R}_{A}$ and $\left\{x \in \mathbb{R}_{A}:(\exists y \in A)(d(x, y)=0)\right\}=A$. Since $A$ is not a zero-set in $\mathbb{R}_{A}, A$ does not satisfy $(a)_{\omega}$ in $\mathbb{R}_{A}$ (see also [18, Corollary 5 to Theorem 1]).

EXAmple 3.5. Under $C H$, there exist sets $A$ and $X$ with $A \subseteq X \subseteq$ $\mathbb{R}$ such that $A$ satisfies $\left(b_{2}\right)$ in $X_{A}$ but fails to satisfy $\left(b_{1}\right)$ in $X_{A}$. Hence, $A$ is $P($ point-finite $)$-embedded in $X_{A}$, but $A \times[0,1]$ is not $P^{\omega}$ (point-finite)embedded in $X \times[0,1]$.

Proof. By [10, Corollary to Lemma 39.4], there exists an analytic set $B$ in $\mathbb{R}$ such that $\mathbb{R} \backslash B$ is not analytic. Put $A=\mathbb{R} \backslash B$ and let $\mathcal{B}$ be the family of all Borel sets in $\mathbb{R}$ containing $A$. Since $|\mathcal{B}|=2^{\omega}$, we can enumerate $\mathcal{B}$ as $\left\{B_{\alpha}: \alpha<\omega_{1}\right\}$ by CH. Then $\bigcap_{\beta<\alpha} B_{\beta} \cap B$ is uncountable for each $\alpha<\omega_{1}$, because $A$ is not a Borel set. Thus, we can choose inductively a point

$$
x_{\alpha} \in\left(\bigcap_{\beta<\alpha} B_{\beta} \cap B\right) \backslash\left\{x_{\beta}: \beta<\alpha\right\}
$$

for each $\alpha<\omega_{1}$. Put $X=A \cup\left\{x_{\alpha}: \alpha<\omega_{1}\right\}$. Then, since $X \backslash B_{\alpha}$ is countable for each $\alpha<\omega$, it follows from Proposition 3.2(2) that $A$ satisfies $\left(b_{2}\right)$ in $X_{A}$. On the other hand, since $B$ is an analytic set in $\mathbb{R}$ and $B_{\alpha} \cap B \neq \emptyset$ for each $\alpha<\omega_{1}$, Proposition 3.2(1) shows that $A$ does not satisfy $\left(b_{1}\right)$ in $X_{A}$.

REMARK 3.6. Let $X_{A}$ be the space defined in Example 3.5, and let $\Omega=\omega_{1}+1$ with the usual order topology. Now, by proving that $A \times \Omega$ is $P$ (point-finite)-embedded in $X_{A} \times \Omega$, we show that the assumption of metrizability of $Y$ is essential in condition (3) of Theorem 2.6. By Lemma 2.2, $A \times \Omega$ is $P$-embedded in $X_{A} \times \Omega$. Thus, by Theorem 2.1, it suffices to show that $A \times \Omega$ satisfies $\left(b_{3}\right)$ in $X_{A} \times \Omega$. Take a countable family $\left\{G_{n}: n<\omega\right\} \subseteq$ $\mathcal{C} o z\left(X_{A} \times \Omega\right)$ with $\bigcap_{n<\omega} G_{n} \cap(A \times \Omega)=\emptyset$. Put $A_{n}=\left\{x \in A:\left\langle x, \omega_{1}\right\rangle \notin G_{n}\right\}$ for each $n<\omega$. Since each $A_{n}$ is separable and each $G_{n}$ is an $F_{\sigma}$-set, we can 
find $\alpha<\omega_{1}$ such that $G_{n} \cap\left(A_{n} \times(\Omega \backslash \alpha)\right)=\emptyset$ for each $n<\omega$. Here, we may assume that $\alpha$ is an isolated ordinal. For each $n<\omega$, put

$$
H_{n}=\operatorname{pr}_{X_{A}}\left[G_{n} \cap\left(X_{A} \times(\Omega \backslash \alpha)\right)\right] .
$$

Then $H_{n} \in \mathcal{C} O z\left(X_{A}\right)$ by Lemma $2.4(2)$, and $\bigcap_{n<\omega} H_{n} \cap A=\emptyset$ as $H_{n} \cap A_{n}=\emptyset$ for each $n<\omega$. Since $A$ satisfies $\left(b_{3}\right)$ in $X_{A}$, there exists $U \in \mathcal{C} o z\left(X_{A}\right)$ such that $\bigcap_{n<\omega} H_{n} \subseteq U$ and $U \cap A=\emptyset$. On the other hand, since $\alpha$ is countable compact metrizable, it follows from Corollary 2.3 and Theorem 2.1 that there exists $V \in \operatorname{Coz}\left(X_{A} \times \alpha\right)$ such that $\bigcap_{n<\omega} G_{n} \cap\left(X_{A} \times \alpha\right) \subseteq V$ and $V \cap(A \times \alpha)=\emptyset$. Finally, putting $W=(U \times(\Omega \backslash \alpha)) \cup V$, we obtain a cozero-set $W$ in $X_{A} \times \Omega$ such that $\bigcap_{n<\omega} G_{n} \subseteq W$ and $W \cap(A \times \Omega)=\emptyset$. Hence, $A \times \Omega$ satisfies $\left(b_{3}\right)$ in $X_{A} \times \Omega$.

Example 3.7. Under $C H$, there exist sets $A$ and $X$ with $A \subseteq X \subseteq \mathbb{R}$ such that $A$ satisfies $\left(b_{3}\right)$ in $X_{A}$ but fails to satisfy $\left(b_{2}\right)$ in $X_{A}$.

Proof. Following [10], $\Sigma_{3}^{0}$ denotes the family of all sets which can be written as the union of countably many $G_{\delta}$-sets in $\mathbb{R}$, and $\Pi_{4}^{0}$ denotes the family of all sets which can be written as the intersection of countably many members of $\Sigma_{3}^{0}$. By [10, Corollary to Lemma 39.1] there exists a Borel set $A$ in $\mathbb{R}$ such that $A \notin \Pi_{4}^{0}$. Now, let $\mathcal{B}$ be the family of all members of $\Pi_{4}^{0}$ containing $A$. Since $|\mathcal{B}|=2^{\omega}$, we can enumerate $\mathcal{B}$ as $\left\{B_{\alpha}: \alpha<\omega_{1}\right\}$ by $\mathrm{CH}$. Then $\bigcap_{\beta<\alpha} B_{\beta} \backslash A$ is uncountable for each $\alpha<\omega_{1}$, because $A \notin \Pi_{4}^{0}$. Hence, we can define a set $X=A \cup\left\{x_{\alpha}: \alpha<\omega_{1}\right\}$ similarly to the proof of Example 3.5. Since $X \backslash B_{\alpha}$ is countable for each $\alpha<\omega_{1}$, it follows from Proposition 3.2(3) that $A$ satisfies $\left(b_{3}\right)$ in $X_{A}$. On the other hand, $\mathbb{R} \backslash A$ is a Borel set in $\mathbb{R}$, but $(\mathbb{R} \backslash A) \cap B_{\alpha} \neq \emptyset$ for each $\alpha<\omega_{1}$. Hence, $A$ does not satisfy $\left(b_{2}\right)$ in $X_{A}$ by Proposition $3.2(2)$.

A similar example to Examples 3.5 and 3.7 was constructed by Michael [12] for a countable non- $G_{\delta}$-set $A$ to show that the product of a Lindelöf space $X_{A}$ with $\mathbb{P}$ is not necessarily normal under $\mathrm{CH}$.

In [15, Example 3], Przymusiński and Wage constructed an example of a collectionwise normal space $Z$ having a closed subspace $K$ which is not $P^{\omega}$ (locally finite)-embedded in $Z$. Finally, we show that an $M$-embedded subspace is not necessarily $P^{\omega}$ (locally finite)-embedded by proving the following:

EXAMPlE 3.8. Every closed subspace $A$ of the collectionwise normal space $Z$ of Przymusinski-Wage is $M$-embedded in $Z$.

Proof. The space $Z$ is constructed from a subspace $W$ of Rudin's Dowker space of [17]. All we need to know about $Z$ is that every $G_{\delta^{-}}$set in $W$ is open and that $Z$ is the union of $W$ and another space $Y$, where $W$ is a $G_{\delta^{-}}$set in $Z$ and $Y$ is an open (in $Z$ ) set which is the topological sum of subspaces of $W$. 
From these facts, if a set $G$ is the union of $G_{\delta^{-}}$sets in $Z$, then both $G \cap W$ and $G \cap Y$ are $G_{\delta}$-sets in $Z$, and therefore, $G$ is a $G_{\delta}$-set in $Z$. Now, let $A$ be a closed subspace of $Z$. Since $Z$ is collectionwise normal, it follows from [19, Theorem 5.2] that $A$ is $P$-embedded in $Z$. To show that $A$ satisfies $\left(a_{\gamma}\right)$ in $Z$ for every infinite cardinal $\gamma$, let $\varrho$ be a $\gamma$-separable continuous pseudometric on $Z$. Then the set $L=\{x \in Z:(\exists y \in A)(\varrho(x, y)=0)\}$ is a $G_{\delta^{-}}$set in $Z$ since it is the union of $G_{\delta^{-}}$sets in $Z$. Thus, by the normality of $Z$, there exists a zero-set $F$ in $Z$ such that $A \subseteq F \subseteq L$. Hence, $A$ is $M$-embedded in $Z$.

4. Another application and questions. By AR we mean an absolute retract for the class of metrizable spaces. In [14] Morita proved that a subspace $A$ of a space $X$ is $P^{\gamma}$-embedded in $X$ if and only if for every complete AR $Y$ with $w(Y) \leq \gamma$, every continuous map from $A$ to $Y$ extends continuously over $X$. As another application of Theorem 2.1, we prove the following theorem by a similar argument to the proofs of Morita's theorems in [14] (see also [9, Theorems 2.8 and 2.14]). We now call a metrizable space $X \sigma$-complete if there exist a metric $d$ on $X$, which induces the topology of $X$, and a countable cover $\left\{X_{n}: n<\omega\right\}$ of $X$ such that each $X_{n}$ is a complete subspace of the metric space $(X, d)$.

THEOREM 4.1. Let $A$ be a subspace of a space $X$ and $\gamma$ an infinite cardinal. Then the following are equivalent:

(1) $A$ is $P^{\gamma}$ (point-finite)-embedded in $X$,

(2) for every $\sigma$-complete $\operatorname{AR~} Y$ with $w(Y) \leq \gamma$, every continuous map from $A$ to $Y$ extends continuously over $X$,

(3) for every Banach space $B$ and every convex $F_{\sigma}$-set $Y$ in $B$ with $w(Y) \leq \gamma$, every continuous map from $A$ to $Y$ extends to a continuous map from $X$ to $Y$.

Proof. (1) $\Rightarrow(2)$ : Let $f: A \rightarrow Y$ be a continuous map to a $\sigma$-complete AR $Y$ with $w(Y) \leq \gamma$. We consider $Y$ a metric space having a countable cover by complete subspaces. Then, by Kuratowski-Wojdysławski's theorem (see [9]), there exist a Banach space $B$ and an isometrical embedding $i: Y \rightarrow B$ such that $w(Z) \leq \gamma$, where $Z$ is the convex hull of $i[Y]$. We identify $Y$ and $i[Y]$. Since $A$ is $P^{\gamma}$-embedded in $X$ and $w\left(\operatorname{cl}_{B} Z\right) \leq \gamma, f$ extends to a continuous map $g: X \rightarrow B$ with $g[X] \subseteq \operatorname{cl}_{B} Z$ by Morita's theorem mentioned above. Since $Y$ is an $F_{\sigma}$-set in $B, g^{-1}[Y]$ is a countable union of zero-sets in $X$ such that $A \subseteq g^{-1}[Y]$. Since $A$ is $P^{\gamma}$ (point-finite)-embedded in $X$, it follows from Theorem 2.1 that there exists a continuous function $\varphi: X \rightarrow[0,1]$ such that the set $F=\varphi^{-1}(0)$ satisfies $A \subseteq F \subseteq g^{-1}[Y]$. Consider the diagonal map 


$$
h=g \triangle \varphi: X \rightarrow B \times[0,1]
$$

and let $p, q$ denote the projections of $B \times[0,1]$ onto $B$ and $[0,1]$, respectively. Then $h[F]=h[X] \cap q^{-1}(0)$ is closed in $h[X]$ and $p[h[F]]=g[F] \subseteq Y$. Since $Y$ is an AR, the restriction $\left.p\right|_{h[F]}$ can be extended to a continuous map $p^{*}: h[X] \rightarrow Y$. Then $p^{*} \circ h: X \rightarrow Y$ is a continuous extension of $\left.(p \circ h)\right|_{A}=\left.g\right|_{A}=f$.

The implication $(2) \Rightarrow(3)$ follows from the fact that every convex $F_{\sigma}$-set in a Banach space is a $\sigma$-complete AR. For a set $S$, let $\ell_{1}(S)$ be the Banach space of all real-valued functions $v$ on $S$ such that $\|v\| \equiv \sum_{s \in S}|v(s)|<\infty$, and $\Delta_{S}$ the subspace of $\ell_{1}(S)$ consisting of all $v \in \ell_{1}(S)$ such that $v(s)=0$ for all but finitely many $s \in S, v \geq 0$, and $\sum_{s \in S} v(s)=1$. Dydak [3] proved that $A$ is $P^{\gamma}$ (point-finite)-embedded in $X$ if (and only if) for every set $S$ with $|S| \leq \gamma$, every continuous map from $A$ to $\Delta_{S}$ extends to a continuous map from $X$ to $\Delta_{S}$. Since $\Delta_{S}$ is a convex $F_{\sigma}$-set in $\ell_{1}(S)$, we have the final implication $(3) \Rightarrow(1)$.

REMARK 4.2. By Hausdorff's extension theorem, a metrizable space is $\sigma$-complete if and only if it has a countable cover by closed completely metrizable subspaces. The term " $\sigma$-complete" was used by A. H. Stone in $[20$, Lemma 4] without an explicit definition.

We conclude the paper with some open questions.

QUESTION 4.3. Does there exist an example in ZFC of a P-embedded subspace which satisfies $\left(b_{3}\right)$ but not $\left(b_{2}\right)$ ? Does there exist an example in ZFC of a P-embedded subspace which satisfies $\left(b_{2}\right)$ but not $\left(b_{1}\right)$ ?

The next question was first asked by the second author in [22, Problem 2.3.4], which asks if there is a $P^{\gamma}$ (locally finite)-embedding analogue of Theorem 2.1.

QUESTION 4.4. Let $A$ be a subspace of a space $X$ and $\gamma$ an uncountable cardinal. Is then $A P^{\gamma}\left(\right.$ locally finite)-embedded in $X$ if $A$ is $P^{\gamma}$-and $P^{\omega}$ (locally finite)-embedded in $X$ ?

Acknowledgments. The authors wish to thank the referee for his/her careful reading and many valuable suggestions which have greatly improved the paper. In particular, they learned from the referee that every Suslin$\mathcal{Z}$-set can be expressed as the inverse image of an analytic subset of the Hilbert cube (Lemma 2.5). This fact enabled them to make the original proofs of Theorem 2.6 and Proposition 2.8 very short and clear. Moreover, the statement in Example 3.8 that every closed subspace of $Z$ is $M$-embedded in $Z$ is due to the referee. In the previous version, the authors proved that only the subspace $K$ is $M$-embedded in $Z$. 


\section{References}

[1] R. A. Alò and L. I. Sennott, Collectionwise normality and the extension of functions on product spaces, Fund. Math. 76 (1972), 231-243.

[2] R. Arens, Extension of coverings, of pseudometrics, and of linear-space-valued mappings, Canad. J. Math. 5 (1953), 211-215.

[3] J. Dydak, Extension theory: the interface between set-theoretic and algebraic topology, Topology Appl. 74 (1996), 225-258.

[4] -, Partitions of unity, Topology Proc. 27 (2003), 125-171.

[5] R. Engelking, General Topology, rev. ed., Heldermann, Berlin, 1989.

[6] Z. Frolík, The topological product of two pseudocompact spaces, Czechoslovak Math. J. 10 (1960), 339-349.

[7] T. E. Gantner, Extensions of uniformly continuous pseudometrics, Trans. Amer. Math. Soc. 132 (1968), 147-157.

[8] L. Gillman and M. Jerison, Rings of Continuous Functions, Van Nostrand, Princeton, 1960.

[9] T. Hoshina, Extensions of mappings II, in: K. Morita and J. Nagata (eds.), Topics in General Topology, North-Holland, Amsterdam, 1989, 41-80.

[10] T. Jech, Set Theory, Academic Press, New York, 1978.

[11] A. S. Kechris, Classical Descriptive Set Theory, Springer, New York, 1994.

[12] E. A. Michael, Paracompactness and the Lindelöf property in finite and countable cartesian products, Compos. Math. 23 (1971), 199-214.

[13] J. van Mill, Infinite-Dimensional Topology, Prerequisites and Introduction, NorthHolland, Amsterdam, 1989.

[14] K. Morita, On generalizations of Borsuk's homotopy extension theorem, Fund. Math. 88 (1975), 1-6.

[15] T. C. Przymusiński and M. L. Wage, Collectionwise normality and extension of locally finite coverings, ibid. 109 (1980), 175-187.

[16] C. A. Rogers and J. E. Jayne, K-analytic sets, in: C. A. Rogers et al., Analytic Sets, Academic Press, New York, 1980, 1-181.

[17] M. E. Rudin, A normal space $X$ for which $X \times I$ is not normal, Fund. Math. 73 (1971), 179-186.

[18] L. I. Sennott, On extending continuous functions into a metrizable AE, Gen. Topology Appl. 8 (1978), 219-228.

[19] H. L. Shapiro, Extensions of pseudometrics, Canad. J. Math. 18 (1966), 981-998.

[20] A. H. Stone, Absolute $F_{\sigma}$ spaces, Proc. Amer. Math. Soc. 13 (1962), 495-499.

[21] K. Yamazaki, Extensions of partitions of unity, Topology Proc. 23 (1998), 289-313.

[22] -, Extensions of mappings on product spaces, Ph.D. thesis, Univ. of Tsukuba, 2000.

[23] - $P$ (locally-finite)-embedding and rectangular normality of product spaces, Topology Appl. 122 (2002), 453-466.

[24] - Extending point-finite partitions of unity, Houston J. Math. 29 (2003), 353-359.

Faculty of Education

Shizuoka University

Ohya, Shizuoka 422-8529, Japan

E-mail: echohta@ipc.shizuoka.ac.jp
Institute of Mathematics University of Tsukuba

Ibaraki 305-8571, Japan

E-mail: kaori@math.tsukuba.ac.jp

Received 29 November 2004;

in revised form 16 September 2005 and 14 April 2006 